\title{
Electrical rectification by selective wave-function coupling in small Ag clusters on $\mathrm{Si}(111)-(7 \times 7)$
}

\author{
Shuanglin Hu, ${ }^{1,2}$ Aidi Zhao, ${ }^{2,3}$ Erjun Kan, ${ }^{1,2}$ Xuefeng Cui,,${ }^{1,2}$ Xieqiu Zhang, ${ }^{1,3}$ Fangfei Ming, ${ }^{1}$ Qiang Fu, ${ }^{2}$ \\ Hongjun Xiang, ${ }^{2}$ Jinlong Yang, ${ }^{2, *}$ and Xudong Xiao ${ }^{1,3, \dagger}$ \\ ${ }^{1}$ Department of Physics, The Chinese University of Hong Kong, Shatin, New Territory, Hong Kong, China \\ ${ }^{2}$ Hefei National Laboratory for Physical Sciences at Microscales, University of Science and Technology of China, Hefei, \\ Anhui 230026, China \\ ${ }^{3}$ Department of Physics, The Hong Kong University of Science \& Technology, Hong Kong, China \\ (Received 13 December 2009; revised manuscript received 23 February 2010; published 29 March 2010)
}

\begin{abstract}
A cluster consisting of as few as three $\mathrm{Ag}$ atoms deposited on $\mathrm{Si}(111)-(7 \times 7)$ surface was found to behave as a strong electric rectifier, with a rectification ratio as large as $\sim 150$. Based on first-principles calculations, we found that the wave functions of electronic states at different energies spread out from the surface to different distances. We demonstrated by theoretical simulations and experiments that the observed rectification is a result of coupling the scanning tunneling microscope tip with selective wave functions of the cluster.
\end{abstract}

DOI: $10.1103 /$ PhysRevB.81.115458

PACS number(s): 73.40.Ei, 68.37.Ef, 73.20.Hb, 73.22.-f

\section{INTRODUCTION}

Electronic transport through single molecule or single nanocluster is the foundation of nanoelectronics. Extensive research has been carried out in this direction for various molecules and clusters. ${ }^{1-15}$ Among different functionalities, electric rectification is a basic function that nanodevices must perform. ${ }^{1}$ In modern microelectronics, this is achieved with semiconductor $p$ - $n$ junction or Schottky junction. However, downscaling a conventional $p-n$ or Schottky diode to nanometer size is shown to greatly reduce the rectification ratio $^{16}$ and is thus not an appropriate pathway for fabricating nanosize rectifiers. Alternative schemes using single molecules or clusters, ${ }^{3-6,10-15}$ with sizes $0.5-3 \mathrm{~nm}$, to replace the much bigger $p$ - and/or $n$-doped inorganic semiconductors have been actively investigated. Pioneered by Aviram and Ratner, ${ }^{1,2}$ molecular rectifiers with a $D-\sigma-A$ or $D-\pi-A$ organic molecule connecting an electron donor moiety $(D)$ to an electron acceptor moiety $(A)$ through an insulating saturated $\sigma$ bridge or a twisted $\pi$ bridge have been demonstrated in Langmuir-Blodgett film ${ }^{17}$ and in single molecule device ${ }^{5,6}$ with various organic molecules. Sandwiching a $\mathrm{NC}_{59}$ molecule with an asymmetric electronic density of states (DOS) around the Fermi level in a double tunnel junction geometry ${ }^{14}$ or a Co nanocluster in an asymmetric double tunnel junction ${ }^{15}$ resulted in strong rectification behavior. For phthalocyanine molecules or carbon nanotubes directly placed on conducting surfaces, the asymmetry of their total DOS around Fermi level has also been explored to achieve rectification at a very small scale.,

Electronic wave functions are known to have a spatial distribution. Such a distribution, particularly within the surface plane, has been imaged extensively for different nanosystems by differential conductance $(d I / d V)$ spectroscopy using scanning tunneling microscope (STM) and spectroscopy (STS). ${ }^{18-20}$ Here, we used an STM tip as one electrode and exploited the wave function distribution in the surface normal direction to achieve an electric rectification on a very small Ag cluster deposited on $\mathrm{Si}(111)-(7 \times 7)$ surface. Firstprinciples calculations indeed show that the wave functions at different eigenenergies for $\mathrm{Ag}$ trimer on $\mathrm{Si}(111)-(7 \times 7)$ spread out to different distance in the surface normal direction. By coupling the STM tip with selected wave functions, strong asymmetric conductance through the Ag nanocluster, i.e., electric rectification, can be realized.

\section{EXPERIMENT AND SIMULATION}

Our experiment was performed in an Omicron ultra-highvacuum system with a base pressure better than 1 $\times 10^{-10}$ torr. One $p$-type $\mathrm{Si}(111)$ substrate (doping density $N_{A}=2.3 \times 10^{15} \mathrm{~cm}^{-3}$, from Virginia Semiconductor Inc., USA $)$ and one $n$-type $\mathrm{Si}(111)$ substrate $\left(N_{D}=1.3\right.$ $\times 10^{16} \mathrm{~cm}^{-3}$, from Institute of Electronic Materials Technology, Poland) were used. The clean and well-reconstructed $\mathrm{Si}(111)-(7 \times 7)$ surface, consisting of the faulted half unit cell (FHUC) and the unfaulted half unit cell (UHUC), was obtained by a routine cleaning procedure. To ensure the cleanness of the substrate, a Si wafer was degassed at $900 \mathrm{~K}$ overnight and then heated to $1200 \mathrm{~K}$ followed by repeated cycles of flashing to $1500 \mathrm{~K}$ for $30 \mathrm{~s}$. A shorter time of $10 \mathrm{~s}$ was used in the last flash to guarantee the quality of a wellreconstructed $(7 \times 7)$ surface. A small amount of $\mathrm{Ag}, \sim 0.04$ monolayer, was then deposited on the clean $\operatorname{Si}(111)-(7 \times 7)$ surface at room temperature by e-beam evaporation. The STM/STS studies were carried out at $78 \mathrm{~K}$ with chemically etched $\mathrm{Pt} / \mathrm{Ir}$ tips, which were cleaned by $\mathrm{Ar}^{+}$sputtering prior to all experiments.

We performed the first-principles calculations using the VASP code ${ }^{21,22}$ within the framework of density-functional theory (DFT). We employed the projector augmented-wave (PAW) method and the exchange correlation functional with the generalized gradient approximation by Perdew, Burke, and Ernzerhof (PBE-GGA). ${ }^{23,24}$ A repeated-slab geometry was used, with six Si layers separated by a $12 \AA$ vacuum. The unit cell had $298 \mathrm{Si}$ atoms and $49 \mathrm{H}$ atoms, the latter were used to terminate the bottom $\mathrm{Si}$ layer. Only $\Gamma$ point was used for the sampling of the Brillouin zone, due to the large unit cell and limited computing resources. The energy cutoff used in our calculations for the plane-wave basis set is 450 $\mathrm{eV}$. We optimize the positions of the $\mathrm{Ag}$ atoms and the top four Si layers until the forces acting on the atoms are smaller 


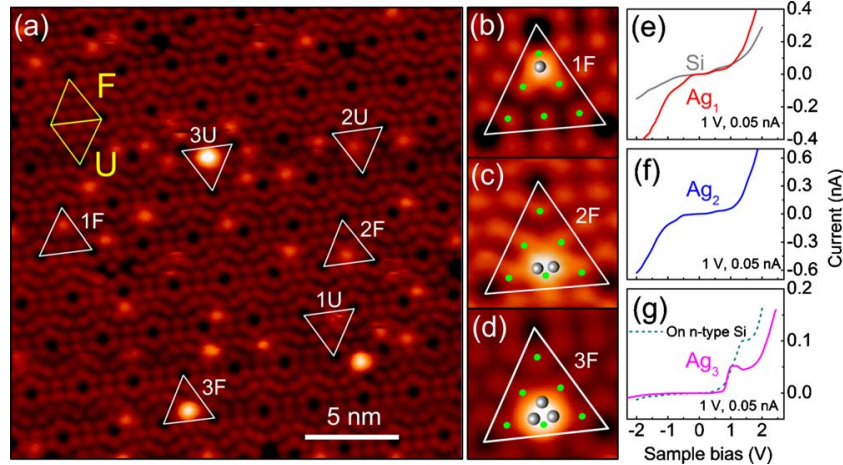

FIG. 1. (Color online) (a) An STM image of Si(111)- $(7 \times 7)$ surface deposited with $\mathrm{Ag}$ atoms taken at $78 \mathrm{~K}$. The image is taken at $V=+1.5 \mathrm{~V}$ and $I=50 \mathrm{pA}$. The typical Ag adsorption features are labeled as $1 \mathrm{~F}, 1 \mathrm{U}, 2 \mathrm{~F}, 2 \mathrm{U}, 3 \mathrm{~F}$, and $3 \mathrm{U}$ according to the number of $\mathrm{Ag}$ atoms in the half unit cell and the type of the half unit cell, where $\mathrm{F}$ and $\mathrm{U}$ represent the faulted and unfaulted half unit cells respectively. (b-d) The close-up STM images for $1 \mathrm{~F}, 2 \mathrm{~F}$, and $3 \mathrm{~F}$ adsorption configurations. The Ag adsorption positions are drawn accordingly. (e-g) The tunneling $I-V$ spectra taken above $\mathrm{Si}$ adatom, $1 \mathrm{~F}, 2 \mathrm{~F}$, and $3 \mathrm{~F}$ features, respectively. All the spectra shown in solid curves are taken on $p$-type silicon substrate. The dashed spectrum in (g) is taken on a $3 \mathrm{~F}$ feature deposited on n-type silicon substrate. All the spectra shown in (e)-(g) are taken at the same set-point of $V=+1.0 \mathrm{~V}$ and $I=50 \mathrm{pA}$.

than $0.02 \mathrm{eV} / \AA$. The energy spectrum for DOS is Gaussian broadened by $0.05 \mathrm{eV}$ and the convergence tolerance of the total energy is $1 \times 10^{-5} \mathrm{eV} /$ unit cell.

\section{RESULTS AND DISCUSSIONS}

In Fig. 1(a), we show an empty-state STM image with Ag atoms adsorbed on a p-type $\mathrm{Si}(111)-(7 \times 7)$ surface at $78 \mathrm{~K}$. Several typical adsorption features, representing respectively the state of one, two, or three $\mathrm{Ag}$ atoms deposited in a half unit cell, were observed in both FHUC and UHUC. Their zoom-in STM images and plausible adsorption configurations are shown in Figs. 1(b)-1(d). By controlling the deposition flux and time, these typical features can be made to appear in sequence, a handle that allowed us to identify the number of $\mathrm{Ag}$ atoms in each typical STM image feature. Single Ag atom can be well identified as a small bright spot (comparable with the image size of a Si adatom) near the corner Si adatoms in the STM images. ${ }^{25}$ The structure shown in Fig. 1(c) is not stable during the STM tip scanning. It can change reversibly into two separate single $\mathrm{Ag}$ atoms [equivalent with Fig. 1(b)] adsorbed within one $7 \times 7$ unit cell. This phenomenon helps us to attribute the structure shown in Fig. 1(c) to a dimer. After increasing the coverage a little more, a larger protrusion structure, as shown in Fig. 1(d), starts to appear both in FHUC and UHUC, which are located symmetrically but near one side of the $7 \times 7$ unit cell. It is attributed to a trimer structure based on its appearance sequence. This typical structure is very stable upon tip scanning and its size, height and cluster position are identical when same bias voltage and set point are used. All the clusters: monomer, dimer and trimer, can adsorb within a half $7 \times 7$ unit cell on

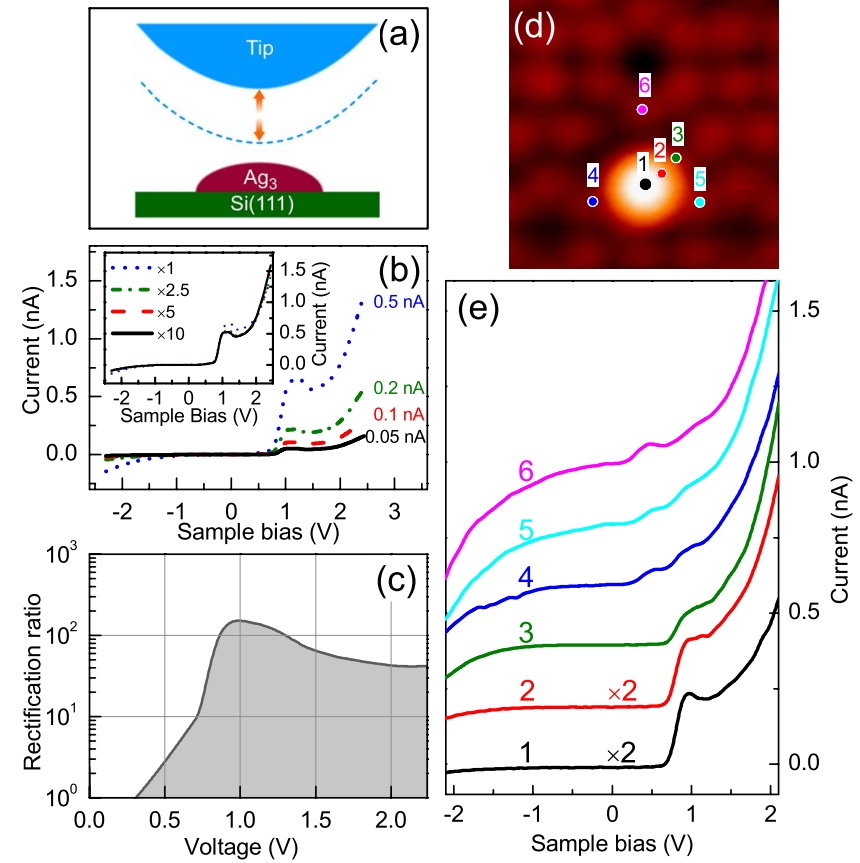

FIG. 2. (Color online) (a) A schematic of the tip- $\mathrm{Ag}_{3}$ tunneling junction with variable junction distances. (b) The tunneling $I-V$ spectra taken at different tip heights over a $3 \mathrm{~F} \mathrm{Ag}$ cluster as set by various tunneling current from 0.05 to $0.5 \mathrm{nA}$ at a sample bias of $+1.0 \mathrm{~V}$. The inset shows the rescaled $I-V$ spectra. (c) The rectification ratio calculated from an $I-V$ curve taken at a tip height set by $V=+1.0 \mathrm{~V}$ and $I=0.1 \mathrm{nA}$. (d) An STM image showing the positions over an $\mathrm{Ag}$ trimer at which the $I-V$ curves are taken. (e) The corresponded $I-V$ spectra from the center of the cluster to the clean $\mathrm{Si}$ adatom, as labeled in (d). The spectra were taken at the tip height set by $V=+1.0 \mathrm{~V}$ and $I=0.1 \mathrm{nA}$ and vertically shifted for clarity.

all equivalent positions with those shown in Figs. 1(b)-1(d). Since the measured tunneling spectra have little dependence on FHUC or UHUC for a given $\mathrm{Ag}$ adsorption configuration, our following discussion will focus on Ag clusters in FHUC only.

Using STS mode, we have measured the tunneling current-voltage $(I-V)$ spectra, as shown by solid curves in Figs. 1(e)-1(g) respectively, for the aforementioned Ag configurations. The STS above a clean Si adatom is also included in Fig. 1(e) for comparison. All the spectra were taken at a tip height set by $+1 \mathrm{~V}$ and $50 \mathrm{pA}$. It is clearly seen that as the number of $\mathrm{Ag}$ atoms increases in the configuration, the $I-V$ curve changes from a more or less symmetric behavior for $\mathrm{Ag}$ monomer and dimer to a strong rectification behavior for Ag trimer. Compared to previously used molecules or nanoclusters consisting of tens of atoms, ${ }^{3-8}$ the $\mathrm{Ag}$ cluster used here consists of as few as three atoms and perhaps represents the smallest electric diode that has ever been reported. ${ }^{26}$

In Fig. 2(b), we depict the $I-V$ curves measured on an $\mathrm{Ag}$ trimer at different tip heights [Fig. 2(a)]. For all of them, the tunneling current is practically zero from negative sample bias up to $+0.7 \mathrm{~V}$. Above the onset voltage at $+0.8 \mathrm{~V}$, the tunneling current increases dramatically and reaches a local maximum at $\sim 1.2 \mathrm{~V}$ before entering into a negative differ- 
ential resistance region. ${ }^{27,28}$ These $I-V$ curves can all be rescaled to collapse together as shown in the inset of Fig. 2(b). In assessing the rectification, the commonly defined rectification ratio (RR), given as the current at a forward bias $V$ divided by the absolute current value at the corresponding reversed bias $-V: \operatorname{RR}(V)=I(V) /|I(-V)|$, was plotted in Fig. 2(c). It takes a value as large as 150 at $+1 \mathrm{~V}$, a quite large number compared to other nanometer size rectifiers, e.g., molecular rectifiers. ${ }^{1}$

We have further investigated the lateral position dependence [Fig. 2(d)] of the rectification by taking a set of $I-V$ spectra at different lateral positions with respect to the $\mathrm{Ag}$ trimer. As can be seen [Fig. 2(e)], the rectification is the strongest near the cluster center, and becomes more and more leaky at negative bias toward the edge of the Ag trimer. Once the tip is off the cluster, the tunneling $I-V$ curves quickly become nonrectifying. Since the $\mathrm{Si}$ adatoms are well resolved in STM images, the effective tip apex is on the order of nanometers. The data here show that the rectification effect is well localized around the Ag trimer, demonstrating a truly nanometer size rectifier.

We can experimentally exclude Schottky effect as the origin, although one might suspect that the rectification from the $\mathrm{Ag}$ trimer is the same as that for a metal-semiconductor junction. As is well known, the Schottky barrier strongly depends on the type of charge carriers in the semiconductor substrate. In our case, when the $p$-type $\mathrm{Si}(111)$ substrate is replaced by a $n$-type $\mathrm{Si}(111)$ substrate, as shown by the dashed curve in Fig. 1(g), the rectification is practically unchanged, without the expected polarity switching. Knowing that the Schottky effect involves a space-charge region and a macroscopic electric field effect, it would render a very localized diode impossible. ${ }^{16}$ The observed well-localized rectification effect for Ag trimer is thus unlikely due to Schottky effect. Furthermore, it would be strange if a Ag trimer and the metallic $\mathrm{Si}(111)-(7 \times 7)$ surface $^{29,30}$ can form a barrier. The finite density of state near the Fermi level as indicated by a finite slope in the measured Si $I-V$ spectrum in Fig. 1(e) is consistent with the metallic nature of the $\operatorname{Si}(111)-(7 \times 7)$ surface.

To investigate the electronic structure of the Ag trimer, we performed the first-principles calculations to obtain the DOS. With a careful optimization, we have found an adsorption configuration [Fig. 3(a)] and simulated its STM image, Fig. 3 (b), which agrees quite well with the observed image [Fig. 3(c)]. The simulation results further confirm our assignment of $\mathrm{Ag}$ trimer based on the appearing sequence.

In Figs. 3(d) and 3(e), the total DOS and the partial DOS (PDOS) for each orbital, i.e., the integrated DOS over the space projected to the respective atomic orbitals of the three $\mathrm{Ag}$ atoms in the $\mathrm{Ag}$ trimer, are shown. Examining the calculated total DOS and the PDOS for each orbital [Fig. 3(d)] for the Ag trimer on $\mathrm{Si}(111)-(7 \times 7)$ surface carefully, we found that there is no distinct asymmetry between the positive and negative energy around the Fermi level either in the total DOS or in the $s, p, d$ PDOS. Thus, a simple-minded picture that our observation is from the electronic DOS of the $\mathrm{Ag}$ trimer cannot hold. However, if we plot the $p_{z}$ and $p_{x+y}$ component of the $p$-PDOS separately, an asymmetry is immediately obvious for the $p_{z}$ component [Fig. 3(e)]. In the nega-

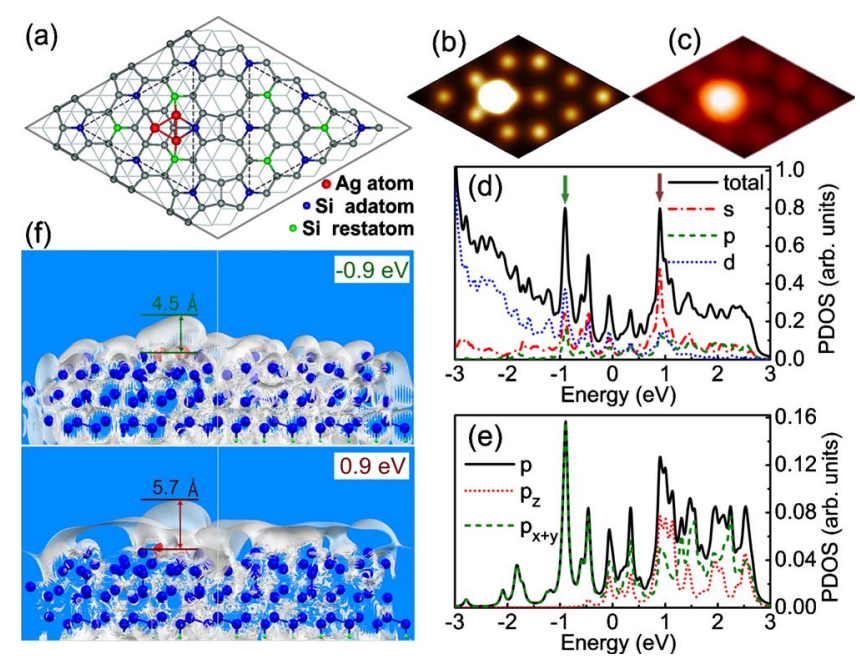

FIG. 3. (Color online) (a) Top view of the optimized computational model for $\mathrm{Ag}_{3} / \mathrm{Si}(111)$ system. (b) The simulated STM image of $\mathrm{Ag}_{3} / \mathrm{Si}(111)$. (c) Experimental STM image of $\mathrm{Ag}_{3}$ on $\mathrm{Si}(111)(+1.0 \mathrm{~V}, 50 \mathrm{pA})$. (d) The total DOS and the $s, p$, and $d$ orbital PDOS for $\mathrm{Ag}_{3} / \mathrm{Si}(111)-(7 \times 7)$. (e) The $p$ orbital PDOS of the $\mathrm{Ag}_{3} / \mathrm{Si}(111)$. The solid black, dotted red, and dashed green lines represent the total $p, p_{z}$, and $p_{x+y}$ component, respectively. (f) The isovalue surfaces of the local density of state of the $\mathrm{Ag}_{3} / \mathrm{Si}(111)$ at $-0.9 \mathrm{eV}$ and at $0.9 \mathrm{eV}$, respectively.

tive energy range, while there are strong $p_{x+y}$ peaks in the PDOS, there is a nearly zero $p_{z}$ PDOS below the Fermi level and a small $p_{z}$ PDOS below $+0.9 \mathrm{eV}$. A strong $p_{z}$ peak around $+0.9 \mathrm{eV}$ roughly locates at the onset position of our experimental $I-V$ curves, $\sim 0.8 \mathrm{~V}$ [Fig. 2(b)]. From the orbital theory, we know that the wave function of out-plane $p_{z}$ orbital may spread out from the surface much more than in-plane $p_{x+y}$ orbitals and provide a possibility for effective wave function coupling between the STM tip and the $p_{z}$ orbital. It is well known that the tunneling current in STS is proportional to the product of tip DOS, sample DOS, and transmission probability. The transmission depends strongly on the tip-sample distance and the geometric distribution of wave function. Only those electronic wave functions with effective overlapping with the tip, rather than the total DOS, can contribute noticeably to tunneling current. ${ }^{31}$ Thus, at a relatively large tip height, the coupling of the tip with the in-plane orbitals $p_{x+y}$ that contribute more to the PDOS in the negative energies is much weaker than that with the outplane $p_{z}$ orbital that contributes more to the PDOS in the positive energies, resulting in a suppression of tunneling current in the negative energy side and thus a strong rectification.

For Ag cluster, a clean and truthful projection of the wave functions into the atomic orbitals is not possible. Thus, to compare the calculated PDOS with the measured $I-V$ curves in STS, we need to know more about the geometric distribution of the local DOS (LDOS). We thus examine the geometric distribution for two representative peaks in the total DOS of $\mathrm{Ag}$ trimer [Fig. 3(d)]. The spatial distribution of the LDOS, one at $-0.9 \mathrm{eV}$ and the other at $+0.9 \mathrm{eV}$, mainly corresponding to these two major peaks in the total DOS of $\mathrm{Ag}$ trimer are shown in Fig. 3(f) with the isovalue 
$0.01 \mathrm{e} / \AA^{3}$. The isovalue surface of LDOS for the energy state at $-0.9 \mathrm{eV}$ is obviously much closer to the surface than that for the energy state at $+0.9 \mathrm{eV}$. When the STM tip is set at a height only well coupled with the states around $+0.9 \mathrm{eV}$, the tunneling current contributed from states around $-0.9 \mathrm{eV}$ would be substantially smaller and a rectification would thus occur. This suggests that the observed rectification originate from the coupling of the STM tip with selected wave functions of the $\mathrm{Ag}$ trimer cluster. In principle, we may also expect such an effect to be operative for Ag monomer and dimmer. Our null experimental observation of rectification for them may come from two reasons: (i) Their $p_{z}$ asymmetry may exist but is weaker due to their strong interaction with the $\mathrm{Si}$ atoms of the substrate. For a Ag trimer, the size and geometric configuration make the interaction among the Ag atoms so strong that it possesses its own electronic structure with less effect from the Si atoms; (ii) The finite contribution to tunneling current from the neighboring $\mathrm{Si}$ atoms, which show no rectification as indicated in Fig. 1(e), around the Ag monomer and dimer due to coupling to the Si wave functions by a finite size tip may render the observed $I-V$ more symmetric.

We also checked the effect of electric field by carrying out calculations with $\pm 0.1 \mathrm{~V} / \AA$ strength perpendicular to the surface. While the electric field do not affect the shape and the peak positions of PDOSs, the isovalue surface of LDOS at both $+0.9 \mathrm{eV}$ and $-0.9 \mathrm{eV}$ are uplifted. The uplift at the positive electric field is somewhat more than that at the negative electric field, although the change of the wave function is insignificant compared to the intrinsic asymmetry in electronic structure. Therefore, the overall effect of electric field in this system is that the asymmetry from coupling with selected wave functions will be enhanced to certain extent.

We confirm our proposed mechanism by simulating the $I-V$ curves at different STM tip heights above the Ag trimer based on Tersoff-Hamann model, ${ }^{32}$ where the tip apex atom is assumed to be a hemisphere with a diameter of $3 \AA$. Use of other apex diameters of $2 \AA, 6 \AA$ and $10 \AA$ leads to similar results. The simulated $I-V$ curves were plotted in Figs. 4(a) and 4(b). When the tip is far away (from $4.5 \AA$ to $3 \AA$ ) from the surface to can only couple with states at positive energy, the simulated $I-V$ curves well reproduce the rectification behavior with a rectification ratio at $2 \mathrm{~V}$ ranges from 5 to 12 [Fig. 4(a)], however, smaller than the experimental data [Fig. 2(b)]. If one considers that the tunneling probability from the occupied states is lower than that from the empty states due to the asymmetry of the tunneling barrier $^{33}$ and the aforementioned electric field effect on the wave function distributions, the current at negative sample bias will become smaller and the agreement with experiment can be improved. The rectification disappears when the tip
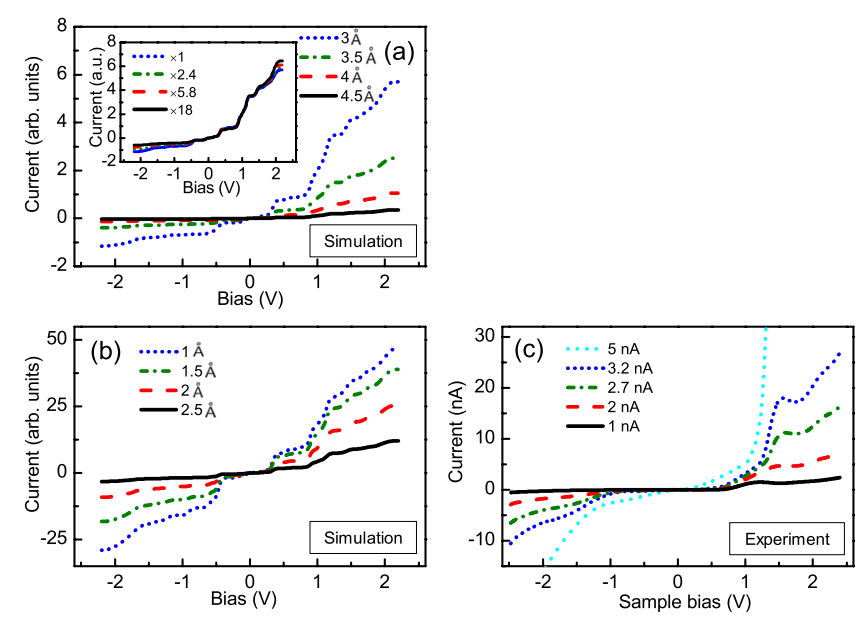

FIG. 4. (Color online) (a) Simulated $I-V$ curves well above the Ag trimer at distances from $3 \AA$ to $4.5 \AA$ higher than the center of $\mathrm{Ag}$ atoms. The inset shows the same curves scaled to the $3 \AA$ one. (b) Simulated $I-V$ curves at distances from $1 \AA$ to $2.5 \AA$ above the center of the Ag trimer. (c) A set of experimental $I-V$ curves measured on the Ag trimer with the set current ranging from 1 to $5 \mathrm{nA}$ at $\mathrm{V}=+1.0 \mathrm{~V}$.

approaches further close to the surface [Fig. 4(b)], which can be explained by the contribution from states at the negative energies which have a closer wave function distribution near the surface. Experimentally, indeed the rectification effect diminishes as the tip-sample separation decreases [Fig. 4(c)]. The good qualitative agreement between the experiments and simulations based on such a simple theoretical framework further supports our proposed rectification mechanism. ${ }^{1-6,10-15,34}$

\section{CONCLUSION}

In summary, we have observed a strong electric rectification behavior from an Ag trimer using scanning tunneling microscope. The theoretical analysis demonstrated that the rectification was originated from a selective coupling between the STM tip and the wave function of the Ag cluster. Changing the tip heights can directly confirm this mechanism. We expect that this mechanism can work not only for electrode in the surface normal direction such as the STM tip but also for electrode fabricated on the surface at an appropriate distance away from the cluster.

\section{ACKNOWLEDGMENTS}

This work was supported by the Research Grants Council of Hong Kong (Grant No. N_CUHK616/06) and the Natural Science Foundation of China (Grant No. 50618001).

\footnotetext{
*Author to whom correspondence should be addressed; jlyang@ustc.edu.cn

†xdxiao@phy.cuhk.edu.hk

${ }^{1}$ See a review, R. M. Metzger, Chem. Rev. 103, 3803 (2003).
}

\footnotetext{
${ }^{2}$ A. Aviram and M. A. Ratner, Chem. Phys. Lett. 29, 277 (1974).

${ }^{3}$ M. Pomerantz, A. Aviram, R. A. Mccorkle, L. Li, and A. G. Schrott, Science 255, 1115 (1992).

${ }^{4}$ P. G. Collins, A. Zettl, H. Bando, A. Thess, and R. E. Smalley,
} 
Science 278, 100 (1997).

${ }^{5}$ J. Chen, M. A. Reed, A. M. Rawlett, and J. M. Tour, Science 286, 1550 (1999).

${ }^{6}$ Z. J. Donhauser, B. A. Mantooth, K. F. Kelly, L. A. Bumm, J. D. Monnell, J. J. Stapleton, D. W. Price, Jr., A. M. Rawlett, D. L. Allara, J. M. Tour, and P. S. Weiss, Science 292, 2303 (2001).

${ }^{7}$ C. Joachim, J. K. Gimzewski, and A. Aviram, Nature (London) 408, 541 (2000).

${ }^{8}$ L. Venkataraman, J. E. Klare, C. Nuckolls, M. S. Hybertsen, and M. L. Steigerwald, Nature (London) 442, 904 (2006).

${ }^{9}$ A. Bannani, C. Bobisch, and R. Moller, Science 315, 1824 (2007).

${ }^{10}$ I. I. Oleynik, M. A. Kozhushner, V. S. Posvyanskii, and L. Yu, Phys. Rev. Lett. 96, 096803 (2006).

${ }^{11}$ S. de Haan, A. Lorke, J. P. Kotthaus, W. Wegscheider, and M. Bichler, Phys. Rev. Lett. 92, 056806 (2004).

${ }^{12}$ J. Taylor, M. Brandbyge, and K. Stokbro, Phys. Rev. Lett. 89, 138301 (2002).

${ }^{13}$ A. Saraiva-Souza, F. M. de Souza, V. F. P. Aleixo, E. C. Girão, J. M. Filho, V. Meunier, B. G. Sumpter, A. G. S. Filho, and J. D. Nero, J. Chem. Phys. 129, 204701 (2008).

${ }^{14}$ J. Zhao, C. G. Zeng, X. Cheng, K. D. Wang, G. W. Wang, J. L. Yang, J. G. Hou, and Q. S. Zhu, Phys. Rev. Lett. 95, 045502 (2005).

${ }^{15}$ A. Iovan, D. B. Haviland, and V. Korenivski, Appl. Phys. Lett. 88, 163503 (2006).

${ }^{16}$ G. D. J. Smit, S. Rogge, and T. M. Klapwijk, Appl. Phys. Lett. 81, 3852 (2002).

${ }^{17}$ A. S. Martin, J. R. Sambles, and G. J. Ashwell, Phys. Rev. Lett. 70, 218 (1993).

${ }^{18}$ L. C. Venema, J. W. G. Wildöer, J. W. Janssen, S. J. Tans, H. L. J. T. Tuinstra, L. P. Kouwenhoven, and C. Dekker, Science 283,
52 (1999).

${ }^{19}$ X. H. Lu, M. Grobis, K. H. Khoo, S. G. Louie, and M. F. Crommie, Phys. Rev. Lett. 90, 096802 (2003).

${ }^{20}$ G. M. Rutter, J. N. Crain, N. P. Guisinger, T. Li, P. N. First, and J. A. Stroscio, Science 317, 219 (2007).

${ }^{21}$ G. Kresse and J. Furthmüller, Phys. Rev. B 54, 11169 (1996).

${ }^{22}$ G. Kresse and J. Hafner, Phys. Rev. B 47, 558 (1993); 49, 14251 (1994).

${ }^{23}$ P. E. Blöchl, Phys. Rev. B 50, 17953 (1994).

${ }^{24}$ J. P. Perdew, K. Burke, and M. Ernzerhof, Phys. Rev. Lett. 77, 3865 (1996).

${ }^{25}$ C. Zhang, G. Chen, K. D. Wang, H. W. Yang, T. Su, C. T. Chan, M. M. T. Loy, and X. D. Xiao, Phys. Rev. Lett. 94, 176104 (2005).

${ }^{26}$ An atomic scale rectification was reported by X. W. Tu et al., J. Chem. Phys. 124, 021105 (2006). However, it is not intrinsic to the atom but rather from the filtering of microwave by the STM preamplifier.

${ }^{27}$ M. Berthe, R. Stiufiuc, B. Grandidier, D. Deresmes, C. Delerue, and D. Stiévenard, Science 319, 436 (2008).

${ }^{28}$ L. Chen, Z. P. Hu, A. D. Zhao, B. Wang, Y. Luo, J. L. Yang, and J. G. Hou, Phys. Rev. Lett. 99, 146803 (2007).

${ }^{29}$ R. J. Hamers, R. M. Tromp, and J. E. Demuth, Phys. Rev. Lett. 56, 1972 (1986).

${ }^{30}$ R. Losio, K. N. Altmann, and F. J. Himpsel, Phys. Rev. B 61, 10845 (2000).

${ }^{31}$ M. Pivetta, F. Silly, F. Patthey, J. P. Pelz, and W.-D. Schneider, Phys. Rev. B 67, 193402 (2003).

${ }^{32}$ J. Tersoff and D. R. Hamann, Phys. Rev. Lett. 50, 1998 (1983).

${ }^{33}$ N. D. Lang, Phys. Rev. B 34, 5947 (1986).

${ }^{34}$ R. M. Feenstra, J. A. Stroscio, and A. P. Fein, Surf. Sci. 181, 295 (1987). 